\title{
La división en lotes: la tensión entre las políticas horizontales y el principio de competencia en la contratación pública
}

\author{
Lucía López Zurita \\ Instituto Universitario Europeo \\ lucia.lopez@eui.eu
}

\section{NOTA BIOGRÁFICA}

Doctoranda en el Instituto Universitario Europeo, Florencia. LLM en Derecho Europeo, Colegio de Europa, Brujas. Principales líneas de investigación: Derecho europeo. Contratación pública.

\section{SUMARIO}

I. INTRODUCCIÓN. II. LA NECESIDAD DE PROMOVER LAS PYMES EN LA UE. III. LA DIVISIÓN EN LOTES COMO POLITICA HORIZONTAL DE PROMOCIÓN DE LAS PYMES. IV. A MODO DE CONCLUSIÓN.

\section{INTRODUCCIÓN}

El artículo 46(1) de la Directiva establece la posibilidad de dividir los contratos públicos en lotes:

«1. Los poderes adjudicadores podrán optar por adjudicar un contrato en forma de lotes separados, y podrán decidir el tamaño y el objeto de dichos lotes.

Excepto en el caso de los contratos cuya división resulte obligatoria en virtud del apartado 4 del presente artículo, los poderes adjudicadores indicarán las principales razones por las cuales han decidido no subdividir en lotes. Dicha decisión se incluirá en los pliegos de la contratación o en el informe específico al que se refiere el artículo 84.

2. Los poderes adjudicadores precisarán, en el anuncio de licitación o en la invitación a confirmar el interés, si las ofertas pueden presentarse para uno, varios o todos los lotes.

Los poderes adjudicadores estarán facultados para limitar el número de lotes que puedan adjudicarse a un solo licitador, incluso en el caso de que se puedan presentar ofertas para varios o todos los lotes, siempre que en el anuncio de licitación o en la invitación a confirmar el interés se indique el número máximo de lotes por licitador. Los poderes adjudicadores indicarán en los pliegos de la contratación los criterios o normas objetivos y no discriminatorios que se proponen aplicar para determinar qué lotes serán adjudicados, en caso de que la aplicación de los criterios de adjudicación pueda dar lugar a que a un solo licitador se le adjudique un número de lotes superior al máximo indicado.

3. Los Estados miembros podrán disponer que, en caso de que pueda adjudicarse más de un lote al mismo licitador, los poderes adjudicadores estén facultados para adjudicar contratos que combinen varios lotes o todos los lotes cuando dichos poderes hayan especificado, en el anuncio de licitación o en la invitación a confirmar el interés, que se reservan dicha posibilidad, y hayan indicado los lotes o grupos de lotes que puedan combinarse».

El precepto introduce una novedad importante, al menos en principio: los poderes adjudicadores parecen obligados a intentar, en la medida de lo posible, que los contratos se dividan en lotes más pequeños. 
Esto no es sino una forma de promoción de las pequeñas y medianas empresas: si los contratos se dividen en lotes más pequeños, las posibilidades de que las PYMES puedan competir por la adjudicación de contratos públicos se incrementan. Ahora bien, la propia Directiva parece entrar en una clara contradicción interna cuando a la vez fomenta la agregación de la demanda a través de la acumulación de lotes (extremo permitido por el propio art. 46) y de la centralización de las compras ${ }^{1}$. Por ello, nos tememos que la división de los contratos en lotes puede tener a la postre unas consecuencias bastante más limitadas de lo que, a primera vista, cabría presumir.

\section{LA NECESIDAD DE PROMOVER LAS PYMES EN LA UE}

Antes de entrar en el análisis del artículo 46.1 de la Directiva, conviene detenerse en un detalle de importancia: la definición de «PYME» no es homogénea en la Unión Europea, sino que varía en cada uno de los Estados Miembros. Sin embargo, un vistazo a la Directiva permite constatar que las referencias a las «PYMES» son constantes. A efectos de la Directiva, la definición de PYME es la que se incluye en la Recomendación de la Comisión de 6 de mayo de $2003^{2}$ (y, como tal, carente de efectos vinculantes): son «PYMES» aquellas empresas que emplean a menos de 250 trabajadores y cuya facturación anual no exceda los 50 millones o que cuyo balance total sea inferior a los 43 millones de euros ${ }^{3}$. Toda referencia a lo largo de este artículo a las PYMES remite a esta definición. Según cálculos de la Comisión, el $99 \%$ de todos los negocios en la Unión son realizados por PYMES ${ }^{4}$. La importancia de estas empresas para la economía de la Unión se hace entonces evidente.

La Comisión también estima que aproximadamente un 16\% del PIB comunitario proviene de la contratación pública ${ }^{5}$. La relevancia de la contratación pública en la economía de la Unión es, por tanto, también indiscutible. Este mercado además tiene la particularidad de estar bajo el control de los Estados, en un doble sentido. En primer lugar, porque son estos quienes deciden qué bienes y servicios necesitan y cómo los necesitan. Y, en segundo lugar, porque lo hacen invirtiendo dinero público. Parece entonces evidente que pueda, o incluso, deba usarse la contratación pública como un modo de promover otros objetivos distintos de los puramente económicos ${ }^{6}$.

La promoción de las pequeñas y medianas empresas es uno de los objetivos fundamentales de la Directiva, lo cual tiene sentido teniendo en cuenta los datos referidos más arriba. En este sentido, la Exposición de Motivos indica con claridad la necesidad de que la regulación de la contratación pública en la Unión sirva para crear una mejor atmósfera para el desenvolvimiento y la competitividad de las PYMES. Ciertamente, son muchas las dificultades a las que las pequeñas y medianas empresas se enfrentan para acceder a los mercados de contratación pública, siendo la principal sus limitadas capacidades económicas y técnicas para concurrir con garantías de éxito a las licitaciones públicas. En este sentido, las excesivas trabas administrativas (puntos 80,84 y 87 de la exposición de motivos) o financieras (punto 83) que padecen las PYMES se contemplan particularmente en la Directiva como problemas específicos de ellas en estos mercados.

Esta capacidad limitada tiene una consecuencia inmediata: para una pequeña o mediana empresa, competir por la licitación de un contrato de gran tamaño entraña una enorme dificultad. Es lógico que contratos de grandes proporciones sobrepasen la capacidad limitada de las PYMES. Por consiguiente, parece también lógico que la división del contrato en lotes más pequeños, tal y como propone el artículo 46.1, puede ser un factor que contribuya a resolver este problema.

Esta medida supone también una novedad en la regulación de los contratos públicos. En la anterior Directiva 2004/18, encontrábamos sólo una mención a la división del contrato en lotes y esta iba referida al cálculo del valor total del contrato (artículo 9). La sospecha que latía tras esta previsión parecía clara: la división

1 Un análisis detallado de la contradicción en I. HERRERA ANCHUSTEGUI, "Division into lots and demand aggregation - extremes looking for the correct balance?", en G. SKOVGAARD OLYKKE, A. SÁNCHEZ GRAELLS (eds.), Reformation or Deformation of the EU Public Procurement Rules, Elgar Edward Publishing, 2016, pág. 125 y ss.

2 Recomendación de la Comisión de 6 de Mayo de 2003, sobre la definición de PYMES (2003/361/EC).

${ }^{3}$ Artículo 2 del Anexo de la Recomendación.

4 Datos de la Comisión en http://ec.europa.eu/growth/smes/business-friendly-environment/sme-definition_en.

${ }_{5}$ http://ec.europa.eu/trade/policy/accessing-markets/public-procurement/.

${ }^{6}$ En este sentido: R. CARANTA, "Sustainable Procurement", en R. CARANTA, M. TRYBUS, G. EDELSTAM (editores), EU Public Contract Law, Bruylant, Bruxelles, 2014, pág. 188. 
del contrato en lotes parecía la manera ideal de evitar la aplicación de la Directiva ${ }^{7}$, puesto que, disminuyendo artificialmente el valor de los contratos, se conseguía que quedasen por debajo del umbral de aplicación de la directiva. No obstante, la medida fue defendida tanto por la Asociación Europea de Pequeñas Empresas (ESBA por sus siglas en inglés) ${ }^{8}$, como por la propia Comisión Europea ${ }^{9}$. Sin embargo no parece que la desconfianza hacia la división en lotes haya cambiado excesivamente entre las autoridades administrativas.

Es muy probable que la razón por la que esta división en lotes no se haga obligatoria en la Directiva resida en esta desconfianza. De todos modos, podría estimarse que la división en lotes posiblemente sea la medida más apropiada que puede adoptarse para mejorar la competitividad y las posibilidades de las PYMES en estos ámbitos sin entrar en colisión con el principio de no discriminación, habida cuenta el recelo existente en la Unión acerca del acogimiento de otras medidas de «discriminación positiva» hacia las PYMES ${ }^{10}$.

\section{LA DIVISIÓN EN LOTES COMO POLÍTICA HORIZONTAL DE PROMOCIÓN DE LAS PYMES}

El artículo 46.1 persigue entonces el objetivo claro de promocionar las PYMES en el mercado único. Todos esos objetivos distintos de los puramente económicos se engloban dentro de las denominadas políticas secundarias, públicas u horizontales ${ }^{11}$. Estos vienen reconocidos en el artículo 67 de la Directiva, al señalar que la oferta económicamente más ventajosa podrá tener en cuenta la relación calidad-precio «que se evaluará en función de criterios que incluyan aspectos cualitativos, medioambientales y/o sociales vinculados al objeto del contrato público de que se trate».

En este sentido, parece evidente que la promoción de las PYMES entra dentro de lo que tradicionalmente se han denominado "políticas industriales» ${ }^{12}$, frente a las sociales y las medioambientales. Curiosamente, son precisamente las políticas industriales las más polémicas, por levantar dudas sobre su compatibilidad con el mercado interno ${ }^{13} \mathrm{y}$, pese a ello, y como ya hemos señalado con anterioridad, la Comisión ha hecho una defensa cerrada de la necesidad de promocionar a las PYMES a través de la contratación pública. Más aún, en un mercado interno que se basa en la no discriminación, pueden surgir dudas razonables sobre hasta qué punto un artículo que obliga a la división del contrato en lotes no conlleva necesariamente un cierto elemento discriminatorio y de distorsión de la competencia ${ }^{14}$. El problema puede ser más teórico que real, dado que la propia Directiva impone serias limitaciones a la posibilidad de división de los contratos.

La primera, y más evidente, es la ya comentada de que la división de contratos no es en caso alguno obligatoria, sino que se entiende sólo como una recomendación a los poderes adjudicadores. Se les impone sólo una obligación de motivar la falta de división en lotes de los contratos ${ }^{15}$. Nótese que el artículo

7 El Tribunal de Cuentas en su informe sobre el sector de la Contratación Pública en 2014 señalaba este como uno de los problemas a tener en cuenta.

8 ESBA de hecho recogió toda una serie de propuestas para favorecer a las PYMES en su informe a la propuesta de Directiva (disponible en inglés aquí: http://www.esba-europe.org/pdf/SubSubSection-163.pdf). Entre ellas, destacaba especialmente la división en lotes de los contratos.

9 European Code of Best Practices Facilitating Access by SMEs To Public Procurement Contracts.

10 La Comisión fue tajante en el Libro Verde para la Modernización del Sector de la Contratación pública: «the EU is not in favor of reserving markets to certain undertakings». Otra medida descartada fue la constitución de "sets-aside», que habría supuesto la reserva de ciertos contratos exclusivamente para las PYMES. Esta opción es común en los Estados Unidos, donde la Small Business Act reconoce esta opción. En la Unión está generalmente prohibida, pero nótese que algunas agencias europeas, al margen de la directiva, tienen algo similar a los sets-aside. Es el caso de la Agencia Espacial Europea. Vid. N. DIMITRI y G. PIGA (editores), Handbook of Public Procurement, Cambridge, 2011, pág. 285.

11 El término «políticas secundarias» parece indicar que este objetivo es adyacente o subordinado al objetivo primario de adquirir bienes y servicios, lo que da lugar a una jerarquía de objetivos que no resulta pertinente (una discusión en BENEDICT, Sekundärzwecke im Vergabeverfahren, Springer, Max-Planck-Institut, Heidelberg 2000, págs. 17-20). Parece, por tanto, que «políticas horizontales» es un mejor modo de referirse a todos estos objetivos distintos de los puramente económicos que pueden perseguirse a través de la contratación pública. Para una discusión detallada, vid. S. ARROWSMITH, "Horizontal Policies in Public Procurement. A taxonomy", Journal of Public Procurement, vol. 10, número 2.

12 CARANTA, no obstante, parece considerarlas como un grupo separado dentro de las políticas horizontales. R. CARANTA/M. TRYBUS y G. EDELSTAM (editores), EU Public Contract Law, pág. 165. pág. 190

13 En este sentido, MORETTINI en E. CHITTI y B. MATARELLA (eds.), Global Administrative Law and EU Administrative Law,

14 Vid. S. ARROWSMITH, "An assessment of the new legislative package on public procurement", Common Market Law Review 41, 2004, 1.277-1.325, pág. 1.315.

15 Es el denominado «decide or explain» principle. C. KONINCK, T. RONSE, W. TIMMERMANS, European Public Procurement Law: The Public Sector Procurement Directive 2014/24/EU explained through 30 Years of Jurisprudence by the Court of Justice of the 
obliga solo a «incluir una indicación» de «las principales razones que expliquen la decisión hecha [sic] por el poder adjudicador». No parece excesivamente oneroso para tal poder adjudicador que, en todo caso, tiene un deber general de motivar sus decisiones. No hay en esta necesidad de motivación nada nuevo. El punto 78 de la Exposición de Motivos enumera incluso algunas de las razones que pueden ser invocadas para justificar la improcedencia de dividir el contrato en lotes: "estas razones podrían ser, por ejemplo, el hecho de que el poder adjudicador considere que dicha división podría conllevar el riesgo de restringir la competencia, o hacer la ejecución del contrato excesivamente difícil u onerosa desde el punto de vista técnico, o que la necesidad de coordinar a los diferentes contratistas para los diversos lotes podría conllevar gravemente el riesgo de socavar la ejecución adecuada del contrato». Leído en conjunción con el artículo 46, parece que la obligación de explicar la falta de división queda reducida a la mínima expresión. La trasposición de la Directiva en la legislación española no parece exigir una mayor justificación. El artículo 99.3 de la nueva Ley de Contratos del Sector Público incluye una lista de motivos que se considerarán "válidos en todo caso» a los efectos de justificar la no división en lotes de un contrato. La presunción iuris et de iure así establecida ahonda más si cabe en la relativa laxitud de la Directiva en este punto.

Por todo lo expuesto, las decisiones del Tribunal de Justicia de la Unión Europea (TJUE) pueden resultar fundamentales a la hora de concretar y delimitar la extensión misma de este deber de motivación en caso de no división de los contratos. La Carta de Derechos Fundamentales de la Unión recoge en su artículo 41 el derecho a una buena administración y su aplicación a este caso será de indudable relevancia. Más aún, el Tribunal podría incluso decidir que la falta de motivación suficiente conduce a una reducción de los incentivos de los operadores para participar en las licitaciones de contratos públicos, lo que equivaldría a una traba en el ejercicio de las libertades fundamentales en el mercado único ${ }^{16}$.

Resulta también llamativo que no estén en el articulado de la Directiva los criterios para la división en lotes o sobre cómo esta podría llevarse a cabo. Hay sólo una tímida mención en el ya citado punto 78 de la Exposición de Motivos, en el que se hace referencia a la posibilidad de dividir los contratos con base en criterios cuantitativos o cualitativos. Pero, insistimos, lamentablemente, no hay una exposición detallada de estos criterios en el articulado de la Directiva.

Otra limitación importante viene establecida por el fomento de la agregación de oferta que parece promover la Directiva. La centralización de compras del artículo 37 es un claro ejemplo al respecto ${ }^{17}$, aunque, sin salir del mismo artículo 46 , en su apartados $2 .^{\circ}$ y $3 .^{\circ}$, se incluyen limitaciones importantes a la división de los contratos en este sentido. Por un lado, se permite que un mismo licitador pueda concurrir a varios lotes al mismo tiempo, o incluso a todos ellos. En términos prácticos, permitir que un licitador concurra a todos los lotes significa que la supuesta ventaja que representaría la división en lotes para las pequeñas empresas se diluye: no hay mucha diferencia entre un licitador que concurre a un contrato de gran tamaño o a una serie de lotes. Pero, además, el apartado $3 .^{\circ}$ otorga, por otro lado, la posibilidad a los poderes adjudicadores de adjudicar contratos que combinen varios lotes o todos los lotes. El único requisito para poder hacer uso de esta prerrogativa es que dicha potestad esté prevista en los pliegos del contrato.

La consecuencia práctica de lo anterior parece clara: no es particularmente difícil para un poder adjudicador contemplar la división en lotes como una simple formalidad. Para ello, bastaría con que: a) se limitase a justificar el porqué de la no división en lotes; y/o b) procediese a la división en lotes, pero permitiendo a la vez que se presentase una oferta por todos los lotes y contemplando en los pliegos la posibilidad de adjudicar todos los lotes a un mismo licitador ${ }^{18}$. La interpretación que sobre estos aspectos realice el Tribunal de Justicia puede ser fundamental, sobre todo en lo relativo a la relación con el artículo 18 de la Directiva, que consagra el principio de competencia ${ }^{19}$.

European Union, pág. 38. Este punto fue modificado con respecto a la propuesta inicial de la directiva. Ver Enmienda núm. 30 en el Informe del Parlamento sobre la Propuesta de 2011.

16 Vid. I. HERRERA ANCHÚSTEGUI, “Division into lots...", cit., pág. 140 y ss.

17 Con independencia de que nos remitamos a la bibliografía ya detallada en nota 1, hemos de insistir en que la contradicción entre ambas políticas, la promoción de la división en lotes por una parte y la centralización de compras por otra, parece clara.

18 Parece cierto que el artículo 99 de la Ley de Contratos del Sector Público española es algo más restrictiva en este punto, pero no lo suficiente como para de facto forzar la división en lotes.

19 Pese a que es la primera vez que tal principio aparece codificado en una Directiva, este venía ya reconocido con anterioridad por el TJUE. Un análisis del significado del principio de competencia en la Opinión de la Abogada General Stix Hackl en el caso c-247/02. Para un análisis detallado de la relación entre competencia y contratación pública en A. SÁNCHEZ GRAELLS, Public Procurement and the EU Competition Rules, Hart Publishing, 2015. 


\section{A MODO DE CONCLUSIÓN}

La Directiva pone mucho énfasis en la promoción de las PYMES y esto es, sin duda, un acontecimiento a celebrar. Después de todo, son las pequeñas y medianas empresas las que conforman el grueso de la economía de la Unión y no parece lógico que deban quedar fuera de los mercados de la contratación pública. En este sentido, la división de los contratos en lotes de menor tamaño puede representar un punto de inflexión importante en la promoción de las PYMES, que justificaría un eventual aumento de los costes administrativos.

Sin embargo, la Directiva no obliga a realizar tal división en lotes y ni siquiera especifica los criterios sobre los que tal división podría llevarse a cabo. Establecer con cierto detalle cómo y cuándo llevar a cabo una división, ya fuera cuantitativa o cualitativa del contrato en lotes, habría fortalecido la política de promoción de las pequeñas y medianas empresas, incluso para el caso en que no se hubiera dado carácter obligatorio a tal división. Si los poderes adjudicadores tuvieran que motivar su decisión de no dividir en lotes con referencia a ciertos criterios legalmente preestablecidos, su discrecionalidad para no dividir se reduciría, incluso aunque no existiera la obligación de dividir.

No obstante, el artículo 46 de la Directiva, por más que trate de revestirse externamente de una veste obligacional, no parece que, en principio, pase de ser una mera recomendación formulada en unos términos relativamente amplios y generosos. La obligación de motivar la decisión de no dividir en lotes parece mínima e, incluso cuando se procede a la división, la posibilidad de realizar ofertas para todos los lotes y la prerrogativa del poder adjudicador de realizar una adjudicación a una oferta integradora limita seriamente tal división. Así, da la impresión de que para los poderes adjudicadores el artículo 46 impone una mera formalidad.

La interpretación expansiva del artículo parece que solo puede venir de la mano de la jurisprudencia del Tribunal. De un lado, una lectura amplia del deber de motivación, a la luz del principio de transparencia y del derecho a una buena administración, podría imponer una obligación de motivar mucho más estricta. Más aún para el caso de que el Tribunal entendiera que la falta o insuficiencia de la motivación supone de hecho que se coartan las libertades fundamentales de los operadores económicos. De otro lado, el principio de competencia, hoy ya codificado en el artículo 18 de la Directiva, parece encontrar un campo de aplicación ideal en la división del contrato en lotes. 